\title{
Corneal Melting after Collagen Cross-Linking for Keratoconus in a Thin Cornea of a Diabetic Patient Treated with Topical Nepafenac: A Case Report with a Literature Review
}

\author{
Karim Mohamed-Noriega Karla Butrón-Valdez \\ Jeronimo Vazquez-Galvan Jibran Mohamed-Noriega \\ Humberto Cavazos-Adame Jesús Mohamed-Hamsho \\ Department of Ophthalmology, Faculty of Medicine, University Hospital 'Dr. Jose Eleuterio \\ Gonzalez', Autonomous University of Nuevo Leon (UANL), Monterrey, Mexico
}

\section{Key Words}

Corneal collagen cross-linking $\cdot$ Nepafenac $\cdot$ Corneal melting $\cdot$ Nonsteroidal anti-inflammatory drugs · Eye drops · Diabetes

\begin{abstract}
Purpose: To report the case of a 50-year-old woman with diabetes that presented with corneal melting and perforation 6 weeks after collagen cross-linking $(\mathrm{CxL})$ for keratoconus $(\mathrm{KC})$ and postoperative use of nepafenac eye drops, a nonsteroidal anti-inflammatory drug (NSAID). Methods: This is a case report of a patient with diabetes, KC and a thin cornea that had undergone left eye corneal $\mathrm{CXL}$ at a different hospital followed by postoperative use of nepafenac eye drops for 6 weeks. Results: The patient presented for the first time to our clinic with left corneal melting, perforation and iris prolapse 6 weeks after corneal $C x L$ and topical nepafenac use. She was treated with a left eye tectonic penetrating keratoplasty, extracapsular cataract extraction, intraocular lens implantation and pupilloplasty. Conclusions: The corneal melting and perforation in this patient was associated with multiple risk factors: (1) nepafenac eye drop use, (2) CxL in a cornea thinner than $400 \mu \mathrm{m}$ and (3) diabetes. The recommended corneal thickness limits should be respected. Topical NSAIDs should be used with caution if used as postoperative treatment after corneal CXL and in patients with diabetes, epithelial defect or delayed healing, because of the possible increased risk for corneal melting when multiple risk factors are observed.

\section{KARGER}


Mohamed-Noriega et al.: Corneal Melting after Collagen Cross-Linking for

Keratoconus in a Thin Cornea of a Diabetic Patient Treated with Topical Nepafenac

\section{Introduction}

Keratoconus (KC) is a noninflammatory corneal ectasia that usually begins at puberty, progresses at a variable rate and often stabilizes with aging. Available treatments include spectacles, rigid gas-permeable contact lenses, intrastromal corneal ring segments, penetrating keratoplasty (PKP) and deep anterior lamellar keratoplasty. All of these treatments aim to improve visual acuity. Corneal collagen cross-linking $(\mathrm{CxL})$ is a new treatment option, and the only one that attempts to halt KC progression $[1,2]$. Corneal CxL uses riboflavin (vitamin $\mathrm{B}_{2}$ ) and ultraviolet $A$ irradiation to enhance corneal rigidity and strength by generating reactive oxygen species that increase chemical covalent bonds between the collagen fibrils $[1,3]$. Topical nepafenac, a nonsteroidal anti-inflammatory drug (NSAID), is a potent cyclooxygenase inhibitor that decreases prostaglandin synthesis [4] and leukotriene [5]. Topical NSAIDs are used to reduce inflammation and pain after ocular surgery $[4,5]$. It is known that diabetes produces delayed wound healing, diabetic keratopathy and increased matrix metalloproteinase (MMP) levels in the cornea [6]. Noninfective corneal melting or keratolysis is characterized by the initial development of an epithelial defect as well as the release of MMP in the cornea and tears. MMP participates in corneal collagen proteolysis and subsequent corneal thinning that may lead to descemetocele formation and corneal perforation [7].

Topical NSAIDs have been associated with corneal melting and perforation after photorefractive keratectomy [4], laser-assisted in situ keratomileusis, cataract surgery [5] and pterygium surgery as well as in other ocular diseases and procedures $[8,9]$. Few case reports caution us about the association of corneal melting after corneal CxL for KC [3, 10-12]. Herein, we report a case of corneal melting and perforation after corneal CxL for $\mathrm{KC}$ in a diabetic patient with thin corneas and on topical nepafenac.

\section{Case Report}

A 50-year-old Mexican woman with diabetes had undergone left eye corneal CxL for KC at a different hospital. Her preoperative left eye simulated keratometry (K) results were 52.3 $\mathrm{D} \times 34^{\circ}$ and $59.3 \mathrm{D} \times 124^{\circ}$, central corneal thickness was $408 \mu \mathrm{m}$, and the thinnest corneal thickness was $374 \mu \mathrm{m}$ as measured with the Orbscan topographer (fig. 1a). On inquiry by the ophthalmologist that had performed the procedure, corneal CxL had been conducted according to the Dresden protocol [5], using hypotonic riboflavin $0.1 \%$ (Ricrolin-TE' ${ }^{\circledR}$; SOOFT, Montegiorgio, Italy) and the VEGA CBM X-Linker (CSO Ophthalmic, Florence, Italy). Postoperatively, she had been started on topical moxifloxacin $0.5 \% 4$ times daily for 1 week (Vigamoxi ${ }^{\circledR}$; Alcon, Mexico, Mexico), lubricant eye drops 3-hourly for 6 weeks and topical nepafenac $0.1 \%$ (Nevanac ${ }^{\circledR}$; Alcon) 4 times daily for 1 week; however, the patient had kept using it for 5 more weeks without her doctor's approval or supervision.

Six weeks after corneal CxL, she was referred to our ophthalmology department because of the development of a left red eye with discharge, pain and blurred vision. On examination, her left eye revealed hyperemia, paracentral corneal melting $(5 \times 5 \mathrm{~mm})$ with corneal thinning, corneal perforation $(2 \times 2 \mathrm{~mm})$, iris prolapse, and a positive Seidel test (fig. 1b). Her best spectacle-corrected visual acuity was 'counting fingers'. No microorganisms were isolated from corneal scrape cultures and staining. Her glycosylated hemoglobin was $9.1 \%$ and fasting blood glucose was $171 \mathrm{mg} / \mathrm{dl}$. She was diagnosed with left eye corneal melting and perforation associated with corneal $\mathrm{CxL}$, nepafenac eye drop use and poorly controlled diabetes. The patient was advised to immediately discontinue the nepafenac eye drops, to start ciprofloxacin eye drops $0.3 \%$ (Sophixin ${ }^{\circledR}$; Sophia, Guadalajara, Mexico) 4 times daily as well 
Mohamed-Noriega et al.: Corneal Melting after Collagen Cross-Linking for Keratoconus in a Thin Cornea of a Diabetic Patient Treated with Topical Nepafenac

as eye patching. She underwent an emergency tectonic PKP with extracapsular cataract extraction, posterior chamber intraocular lens implantation and pupilloplasty. No complications were observed during the procedure. Histologic evaluation of the excised button revealed fibrosis, chronic inflammation and stroma loss associated with corneal thinning (fig. 1c); no microorganisms were isolated from cultures of the excised cornea. One month after PKP, the tectonic graft was clear, uncorrected visual acuity was $20 / 200$, and pinhole visual acuity was 20/60 (fig. 1d).

\section{Discussion}

We reported a case of corneal melting 6 weeks after corneal CxL for KC in a thin cornea associated with prolonged topical nepafenac use and poorly controlled diabetes that required a tectonic PKP. The proposed multiple risk factors for corneal melting in our patient were prolonged nepafenac eye drop use over a deepithelized cornea, poorly controlled diabetes and corneal CxL in a thinner-than-recommended cornea. Corneal melting is related to an imbalance between extracellular matrix deposition and degradation by proteases including MMPs $[6,7,13]$. An epithelial defect associated with CxL, recurrent corneal erosions and a neurotrophic cornea associated with diabetes and nepafenac eye drop use can induce an upregulation of MMPs in the cornea and are predisposing factors for corneal melting and perforation $[3,4,7,9,13]$.

There are few case reports of corneal melting after corneal CxL for $\mathrm{KC}$ at the time of writing [3, 10-12]. Labiris et al. [10] reported a case of corneal melting after CxL for KC that started with severe inflammation during the first postoperative days with subsequent persistent epithelial defect, corneal melting and perforation 2 months after corneal CxL without any other predisposing factor. Faschinger et al. [11] reported a case of bilateral corneal melting after $\mathrm{CxL}$ for $\mathrm{KC}$ after 1 week in the right eye and after 1 month in the left eye of a patient with Down syndrome. They proposed a preoperative stromal thickness of less than $400 \mu \mathrm{m}$ as the predisposing risk factor. Angunawela et al. [12] presented a case of peripheral sterile infiltrate and corneal melting 5 days after corneal CxL for KC. They proposed staphylococcal antigen deposition in areas of static tear pooling beneath the bandage contact lens as the predisposing risk factor. Gokhale and Vemuganti [3] reported a case of corneal melting and perforation 1 week after corneal CxL for KC. They associated the concomitant postoperative use of topical diclofenac and proparacaine eye drops after corneal CxL as the predisposing risk factors.

Corneal CxL has been reported to be effective as an alternative treatment to halt the corneal melting associated with infectious keratitis that does not improve with standard medication [1, 14]. Corneal CxL has a good safety profile [1, 3, 10]; however, there is concern about the induced keratocyte apoptosis, possible endothelial damage to thin corneas and irreversible corneal edema after corneal CxL [1]. Keratocyte damaging after CxL may affect the wound healing process and collagen deposition [1], and the epithelial defect after CxL may increase MMP levels in the cornea and tears [7], therefore increasing the risk for corneal melting. In this report, the stromal corneal thickness was less than $400 \mu \mathrm{m}$, and this is a risk factor for corneal damage after CxL [1]. The currently recommended age range for corneal CxL is 18-40 years. Our patient was 50 years old, and a progressive $\mathrm{KC}$ is unlikely at this age; however, the use and benefit of CxL has been reported for similar age groups [2].

Complications related to NSAID eye drops are infrequent; however, such eye drops have been associated with stinging, irritation, superficial punctate keratitis, epithelial defects and, rarely, corneal infiltrates, corneal melting and corneal perforation $[3,8]$. Predisposing fac- 
tors for NSAID-related side effects include epithelial breakdown, delayed wound healing, autoimmune diseases, diabetes, rosacea and dry eye syndrome [5]. Topical NSAIDs (including nepafenac) can damage the corneal epithelial cytoskeleton, reduce corneal sensitivity [4, 9] and induce an upregulation of MMPs in the cornea and tears [4, 5, 9, 13]. High levels of MMP-2, MMP-3, MMP-8 and MMP-9 have been found in the epithelium and stroma from melted and perforated corneas after topical NSAID (diclofenac or nepafenac) use and in diabetic patients [13].

The occurrence of sterile corneal melting after nepafenac eye drop use is well known but rare; however, the exact mechanism is not fully understood [4, 9]. Feiz et al. [4] reported bilateral corneal melting 4 days after uneventful photorefractive keratectomy and nepafenac eye drop use. Bekendam et al. [9] reported a case of corneal melting and ulceration 3 weeks after orbital decompression surgery for Graves orbitopathy that was started on topical nepafenac eye drops to treat ocular pain associated with an epithelial defect and corneal erosions in a patient with dry eye syndrome, a history of pars plana vitrectomy, cataract extraction and moderately decreased corneal sensation. The corneal melting and ulceration resolved after discontinuation of the nepafenac eye drops. Di Pascuale et al. [15] presented a case of peripheral corneal melting and ulceration 5 months after topical nepafenac use for chronic cystoid macular edema after cataract extraction; the corneal melting resolved after discontinuation of nepafenac.

Diabetes had an additive effect as a risk factor for corneal melting. Corneas of diabetic patients are susceptible to nerve damage, hypoesthesia and subsequent neurotrophic cornea development, delayed corneal wound healing, delayed reepithelization, persistent epithelial defect and increased corneal expression of MMP $[6,7,13]$. Overexpression of MMP-10 seems to be a major contributor to these abnormalities in diabetic patients [6]. The possible additive interaction of diabetes and topical NSAID use in the development of corneal melting has previously been implied [13].

As far as we are aware, this is the first reported case of corneal melting and perforation in a patient with multiple associated risk factors: nepafenac eye drop use, corneal CxL in a thin cornea and diabetes. It is important to prevent complications by properly selecting patients for treatment with either corneal CxL or topical NSAID eye drops, even though they have a good safety profile. The recommended corneal thickness limits for corneal CxL should be respected. Topical NSAIDs should be used with caution as part of postoperative treatment after corneal $\mathrm{CxL}$, as well as in patients with diabetes, epithelial defects or with delayed wound healing because of the increased risk for corneal melting when multiple risk factors are observed.

\section{Acknowledgments}

This case report was critically reviewed and corrected for language clarity and proficiency by professional English-language editing services and native English-speaking professionals in our institutional research vice-directorate, in particular by Sergio LozanoRodriguez, MD.

\section{Statement of Ethics}

This case report was conducted in accordance with good clinical practices and the Declaration of Helsinki. The patient read and provided written informed consent to participate. 
Mohamed-Noriega et al.: Corneal Melting after Collagen Cross-Linking for Keratoconus in a Thin Cornea of a Diabetic Patient Treated with Topical Nepafenac

\section{Disclosure Statement}

The authors declare no conflicts of interest.

\section{References}

1 Gkika M, Labiris G, Kozobolis V: Corneal collagen cross-linking using riboflavin and ultraviolet-A irradiation: a review of clinical and experimental studies. Int Ophthalmol 2011;31:309-319.

-2 Vinciguerra R, Romano MR, Camesasca FI, Azzolini C, Trazza S, Morenghi E, et al: Corneal cross-linking as a treatment for keratoconus: four-year morphologic and clinical outcomes with respect to patient age. Ophthalmology 2013;120:908-916.

-3 Gokhale NS, Vemuganti GK: Diclofenac-induced acute corneal melt after collagen crosslinking for keratoconus. Cornea 2010;29:117-119.

-4 Feiz V, Oberg TJ, Kurz CJ, et al: Nepafenac-associated bilateral corneal melt after photorefractive keratectomy. Cornea 2009;28:948-950.

-5 Wolf EJ, Kleiman LZ, Schrier A: Nepafenac-associated corneal melt. J Cataract Refract Surg 2007;33:19741975.

6 Saghizadeh M, Brown DJ, Castellon R, et al: Overexpression of matrix metalloproteinase-10 and matrix metalloproteinase-3 in human diabetic corneas: a possible mechanism of basement membrane and integrin alterations. Am J Pathol 2001;158:723-734.

7 Sakimoto T, Sawa M: Metalloproteinases in corneal diseases: degradation and processing. Cornea 2012;31(suppl 1):S50-S56.

8 Flach AJ: Corneal melts associated with topically applied nonsteroidal anti-inflammatory drugs. Trans Am Ophthalmol Soc 2001;99:205-210; discussion 210-212.

$>9$ Bekendam PD, Narváez J, Agarwal M: Case of corneal melting associated with the use of topical nepafenac. Cornea 2007;26:1002-1003.

10 Labiris G, Kaloghianni E, Koukoula S, Zissimopoulos A, Kozobolis VP: Corneal melting after collagen crosslinking for keratoconus: a case report. J Med Case Rep 2011;5:152.

-11 Faschinger C, Kleinert R, Wedrich A: Corneal melting in both eyes after simultaneous corneal cross-linking in a patient with keratoconus and Down syndrome (in German). Ophthalmologe 2010;107:951-952, 954955.

12 Angunawela RI, Arnalich-Montiel F, Allan BD: Peripheral sterile corneal infiltrates and melting after collagen crosslinking for keratoconus. J Cataract Refract Surg 2009;35:606-607.

-13 Gabison EE, Chastang P, Menashi S, et al: Late corneal perforation after photorefractive keratectomy associated with topical diclofenac: involvement of matrix metalloproteinases. Ophthalmology 2003;110:1626-1631.

14 Iseli HP, Thiel AM, Hafezi F, et al: Ultraviolet A/riboflavin corneal cross-linking for infectious keratitis associated with corneal melts. Cornea 2008;27:590-594.

15 Di Pascuale MA, Whitson JT, Mootha VV: Corneal melting after use of nepafenac in a patient with chronic cystoid macular edema after cataract surgery. Eye Contact Lens 2008;34:129-130. 
Mohamed-Noriega et al.: Corneal Melting after Collagen Cross-Linking for Keratoconus in a Thin Cornea of a Diabetic Patient Treated with Topical Nepafenac
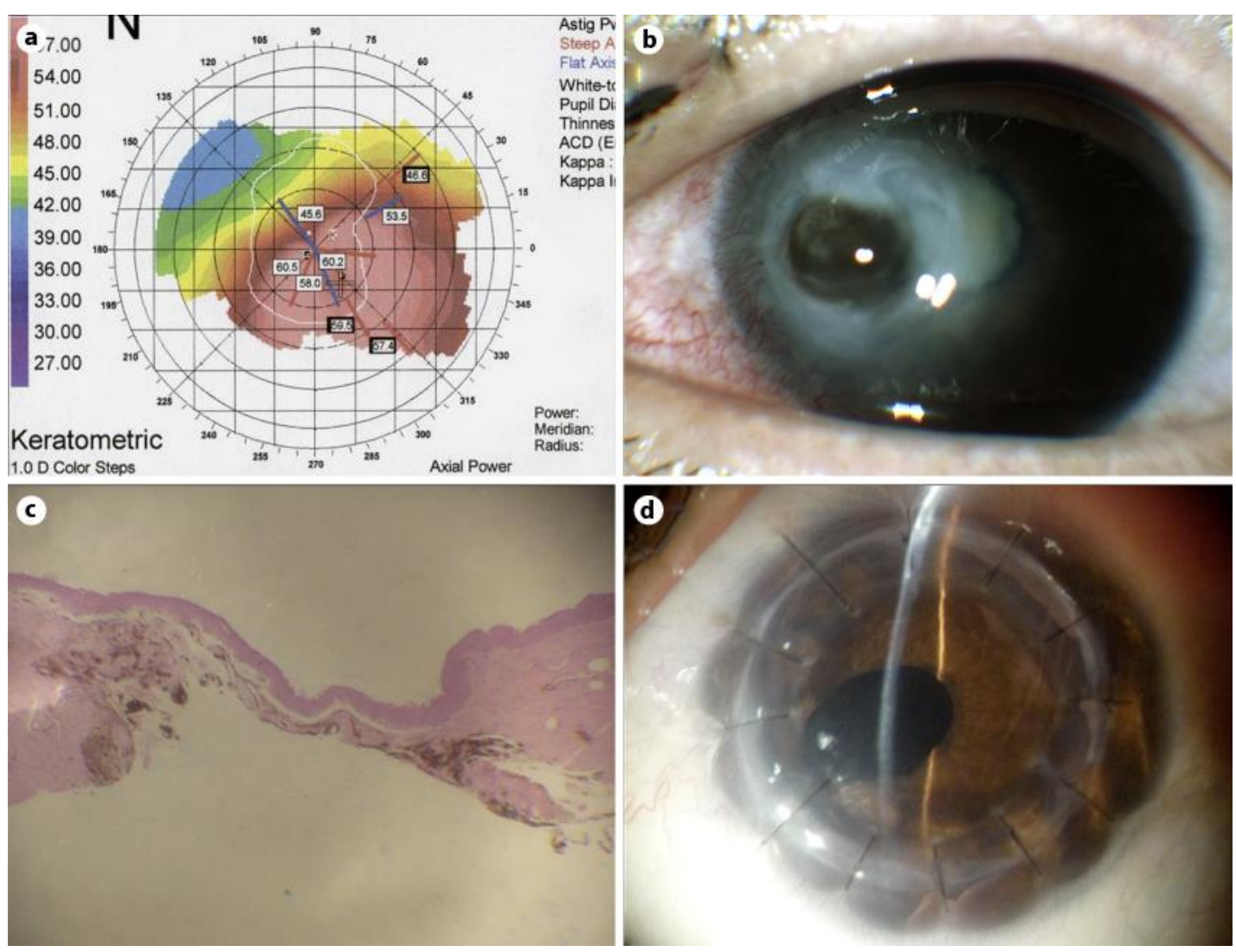

Fig. 1. Corneal melting and perforation after corneal CxL for $\mathrm{KC}$ associated with nepafenac eye drop use in a diabetic patient. a Corneal topography before corneal CxL, showing inferior steepening typical of KC. b Paracentral corneal melting and thinning with a large perforation and iris prolapse 6 weeks after corneal CxL and topical nepafenac use. c Histologic evaluation of the excised cornea revealing inflammatory cells and loss of stroma associated with the corneal melting and pigmented cells associated with iris contact with the cornea. In this section, the epithelium is preserved, because the descemetocele was epithelized in the areas surrounding the perforation and iris prolapse. $\mathbf{d}$ Clear cornea 1 month after successful tectonic PKP, extracapsular cataract extraction, intraocular lens implantation and pupilloplasty. 